OPEN ACCESS

Edited by:

Raffaele Capasso,

University of Naples Federico II,

Italy

Reviewed by:

Oksana Zayachkivska,

Danylo Halytsky Lviv National Medical

University, Ukraine

Ajay Godwin Potnuri,

National Animal Resource Facility for

Biomedical Research (NARFBR),

India

${ }^{*}$ Correspondence: James Cheng-Chung Wei jccwei@gmail.com

Specialty section: This article was submitted to Pharmaceutical Medicine and Outcomes Research,

a section of the journal

Frontiers in Pharmacology

Received: 24 September 2019 Accepted: 17 April 2020 Published: 08 May 2020

Citation:

Wang $Y-T$, Tsai $M-C$, Wang $Y-H$ and Wei JC-C (2020) Association Between Proton Pump Inhibitors and Asthma: A Population-Based Cohort Study.

Front. Pharmacol. 11:607. doi: 10.3389/fphar.2020.00607

\section{Association Between Proton Pump Inhibitors and Asthma: A Population- Based Cohort Study}

\author{
Yao-Tung Wang ${ }^{1,2,3}$, Ming-Chang Tsai ${ }^{1,2,4}$, Yu-Hsun Wang ${ }^{5}$ \\ and James Cheng-Chung Wei ${ }^{1,6,7,8 *}$
}

${ }^{1}$ Institute of Medicine, Chung Shan Medical University, Taichung, Taiwan, ${ }^{2}$ School of Medicine, Chung Shan Medical University, Taichung, Taiwan, ${ }^{3}$ Division of Pulmonary Medicine, Department of Internal Medicine, Chung Shan Medical University Hospital, Taichung, Taiwan, ${ }^{4}$ Division of Gastroenterology and Hepatology, Department of Internal Medicine, Chung Shan Medical University Hospital, Taichung, Taiwan, ${ }^{5}$ Department of Medical Research, Chung Shan Medical University Hospital, Taichung, Taiwan, ${ }^{6}$ Beijing Tsinghua Changgung Hospital, School of Clinical Medicine, Tsinghua University, Beijing, China, ${ }^{7}$ Division of Allergy, Immunology and Rheumatology, Department of Internal Medicine, Chung Shan Medical University Hospital, Taichung, Taiwan, ${ }^{8}$ Graduate Institute of Integrated Medicine, China Medical University, Taichung, Taiwan

Objective: The relationship between proton pump inhibitors (PPIs) and asthma is controversial. The goal of this study was to determine the association between PPI use in non-asthma subjects and their subsequent asthma prevalence.

Design: Nationwide, population-based cohort study.

Methods: We conducted a nationwide, population-based retrospective cohort study using data from the National Health Insurance Research Database (NHIRD) of Taiwan from 1999 to 2013. We identified 24,077 adult patients with PPI use for more than 3 months and 24,077 controls matched by propensity score on a one-to-one ratio for age, gender, comorbidities (hypertension, hyperlipidemia, gastroesophageal reflux disease, allergic rhinitis, atopic dermatitis, peptic ulcer disease, diabetes, and sleep apnea syndrome), and medications (histamine 2 receptor antagonists [H2RA], nonsteroidal anti-inflammatory drugs [NSAIDs], and acetaminophen). The cumulative asthma incidence for the two cohorts in the follow-up period was estimated with the KaplanMeier method, and the difference was examined using the log-rank test. Multivariate Cox regression models were used to calculate the adjusted hazard ratios $(\mathrm{HR})$.

Results: The overall incidence of asthma was 1.58-fold greater in the PPI cohort than in the non-PPI cohort (13.3 versus 8.4 per 1,000 person-years), with an adjusted HR of 1.76 (95\% confidence interval [Cl], 1.64-1.88). In patients without previous peptic ulcer disease, the adjusted HR of asthma associated with PPIs was higher than in the nonPPI group (1.95; 95\% Cl, 1.80-2.11). The risk of asthma due to PPI use was also more significant in patients not receiving H2RA (1.81; 95\% Cl, 1.66-1.96), NSAIDs (1.93; 95\% $\mathrm{Cl}, 1.73-2.15)$, and acetaminophen (1.88; 95\% Cl, 1.70-2.08). 


\section{Conclusions: This population base study demonstrated that patients with long-duration} of PPI use are at a higher risk of developing asthma, regardless of age, gender, comorbidities, and medications.

\section{Keywords: proton pump inhibitors, asthma, National Health Insurance Research Database, population base study,} histamine 2 receptor antagonists

\section{HIGHLIGHTS}

1. What is already known about this topic?

The relationship between proton pump inhibitors (PPIs) and asthma is controversial. Proton pump inhibitors are the most common acid-suppressive medications of GERD which can contribute to asthma symptoms or has been identified as a potential trigger for asthma. Increasing numbers of studies have suggested PPIs are associated with significant rise of adverse health outcomes.

2. What does this article add to our knowledge?

Our data suggest that patients with long-time PPI use have a significantly higher risk of developing asthma when compared with the general population, regardless of gender and age.

3. How does this study impact current management guidelines?

The higher risk of developing asthma of patients with long-duration PPI use is identified based on the general population. We recommended H2RA use prior to PPIs in patients who have no underlying peptic ulcers.

\section{INTRODUCTION}

Clinically, gastroesophageal reflux disease (GERD) is often reported to be a common cause of chronic cough (Kastelik et al., 2005; Irwin et al., 2006). GERD can also contribute to asthma symptoms or has been identified as a potential trigger for asthma (Depla et al., 1988; Pope, 1994; Harding, 2003; Chipps et al., 2018). The cause may be an aspiration of acid or gastric contents into airways and alveoli, which then leads to stimulation of receptors in the lower respiratory tract or chronic inflammation and damage to the alveolar-capillary membrane (Ravelli et al., 2006). GERD and asthma often coexist: GERD has a prevalence ranging from 30 to $90 \%$ in persons with asthma compared to an average of $24 \%$ in non-asthmatic controls (Bor et al., 2010; Broers et al., 2018).

GERD treatments include the use of proton pump inhibitors (PPIs), which are the most common acid-suppressive medications (Forgacs and Loganayagam, 2008) and the firstline antisecretory therapy (Wolfe and Sachs, 2000). PPIs are often used for empiric management of chronic cough with a suspected relationship to GERD (Kahrilas et al., 2016); indeed,

Abbreviations: PPIs, proton pump inhibitors; GERD, gastroesophageal reflux disease; H2RA, histamine 2 receptor antagonists; NSAIDs, nonsteroidal antiinflammatory drugs. two placebo-controlled trials have suggested that PPI treatment relieves GERD-related chronic cough (Harding, 2003). Many studies have been conducted to evaluate and confirm the efficacy of PPI therapy on asthma outcome in patients with asthma with or without GERD. For example, aggressive acid suppressive therapy with omeprazole for 3 months improved asthma symptoms and pulmonary function in $73 \%$ of asthmatics with GERD (Meier et al., 1994).

PPI is a key component in the treatment of chronic cough due to GERD and an optimum regimen to improve asthma symptoms and pulmonary function in select patients. The aim of this study was to identify the association between PPI use in non-asthma subjects and their subsequent asthma prevalence.

\section{METHODS}

\section{Data Resources}

This study is a nationwide, population-based retrospective cohort study based on the Taiwan National Health Insurance Research Database (NHIRD) of one million patients between January1, 1999 and December 31, 2013. The NHIRD, established by the Bureau of National Health Insurance (BNHI) and the National Health Research Institutes (NHRI), included health care data that covered more than $99 \%$ of the Taiwanese population (approximately 23 million people) (https://www. nhi.gov.tw/english/Default.aspx). For patient privacy, the patient identity numbers are encrypted by the NHIRD. The database is a record of all the insurance information, such as medical records, and includes disease diagnosis, drug prescriptions (order date and duration, drug name, dosage, frequency, and administration route), medical procedures, and date of clinic visits and hospitalizations (Lee et al., 2010). The diseases in the NHIRD were defined according to the International Statistical Classification of Diseases and Related Health Problems-9th Edition, clinical modification (ICD-9$\mathrm{CM})$ codes.

This study was approved by the Institutional Review Board (IRB) of Chung Shan Medical University Hospital, Taiwan (CS15134). The IRB waived the need for informed consent for this retrospective study, based on the NHIRD. All protocols were performed in accordance with the relevant guidelines and regulations and were under the surveillance of the IRB of Chung Shan Medical University Hospital.

\section{Study Population}

The new PPI users were selected from one million random samples from the NHIRD between 2000 and 2012 by searching 
for use of the PPI drugs omeprazole, esomeprazole, lansoprazole, pantoprazole, and rabeprazole for more than 90 days and patient age more than twenty years. The index date was operationalized as the first date of PPI use. The outcome of the analysis was a diagnosis of asthma. The asthma cases were included from the same random samples using the codes ICD-9-CM 493.XX and included admitted inpatients or outpatients with at least two visits to the outpatient department clinics. The asthma-related comorbidities of hypertension patients were identified using the ICD-9-CM codes 401-405, and hyperlipidemia was identified with the ICD-9-CM codes 272.0-272.4. Other selected conditions included GERD (ICD-9-CM codes 530.11 and 530.81), allergic rhinitis (ICD-9-CM codes 472, 473 and 477), atopic dermatitis (ICD-9-CM codes 691), peptic ulcer disease (ICD-9-CM codes 531-534.9), diabetes (ICD-9-CM codes 250), and sleep apnea syndrome (ICD-9-CM codes 327.23, 780.51, 780.52, 780.53, and780.57).

In the treatment cohort, 65,291 new PPI users were enrolled, and 64,331 patient samples were obtained after matching to 128,662 non-PPI users in the control group by age and gender in 1:2 ratios (Figure 1). After a follow-up duration of more than ninety days and excluding the asthma cases diagnosed before the index date, 52,389 PPI users in the case group and 113,026 nonPPI patients in the control group were enrolled. Patients who had been diagnosed with asthma prior to the use of PPIs were excluded to ensure that a causal relationship could be analyzed in this study.

The PPI case group and the non-PPI control group also were matched by propensity score on a one-to-one ratio for age, gender, comorbidities (e.g., hypertension, hyperlipidemia, GERD, allergic rhinitis, atopic dermatitis, peptic ulcer disease, diabetes, and sleep apnea syndrome), and medications (e.g., histamine 2 receptor antagonists [H2RA], nonsteroidal antiinflammatory drugs [NSAIDs], and acetaminophen). Ultimately, 24,077 subjects were included in the PPI group and 24,077 in the non-PPI group.

\section{Data Processing and Statistical Analysis}

We used the Chi-square test for categorical variables and t-tests for continuous variables to analyze the differences in the demographic data between the PPI and non-PPI groups. The Kaplan-Meier method was used to estimate the cumulative asthma incidence for the two cohorts in the follow-up period and the log-rank test was used to examine the difference. Multivariate Cox regression models were used to calculate the adjusted hazard ratios (HRs, including the 95\% confidence intervals [CIs]) of the PPI group for non-PPI group after adjusting for age, gender, hypertension, hyperlipidemia, GERD, allergic rhinitis, peptic ulcer disease, diabetes, sleep apnea syndrome, H2RA, NSAIDs, and acetaminophen. All data

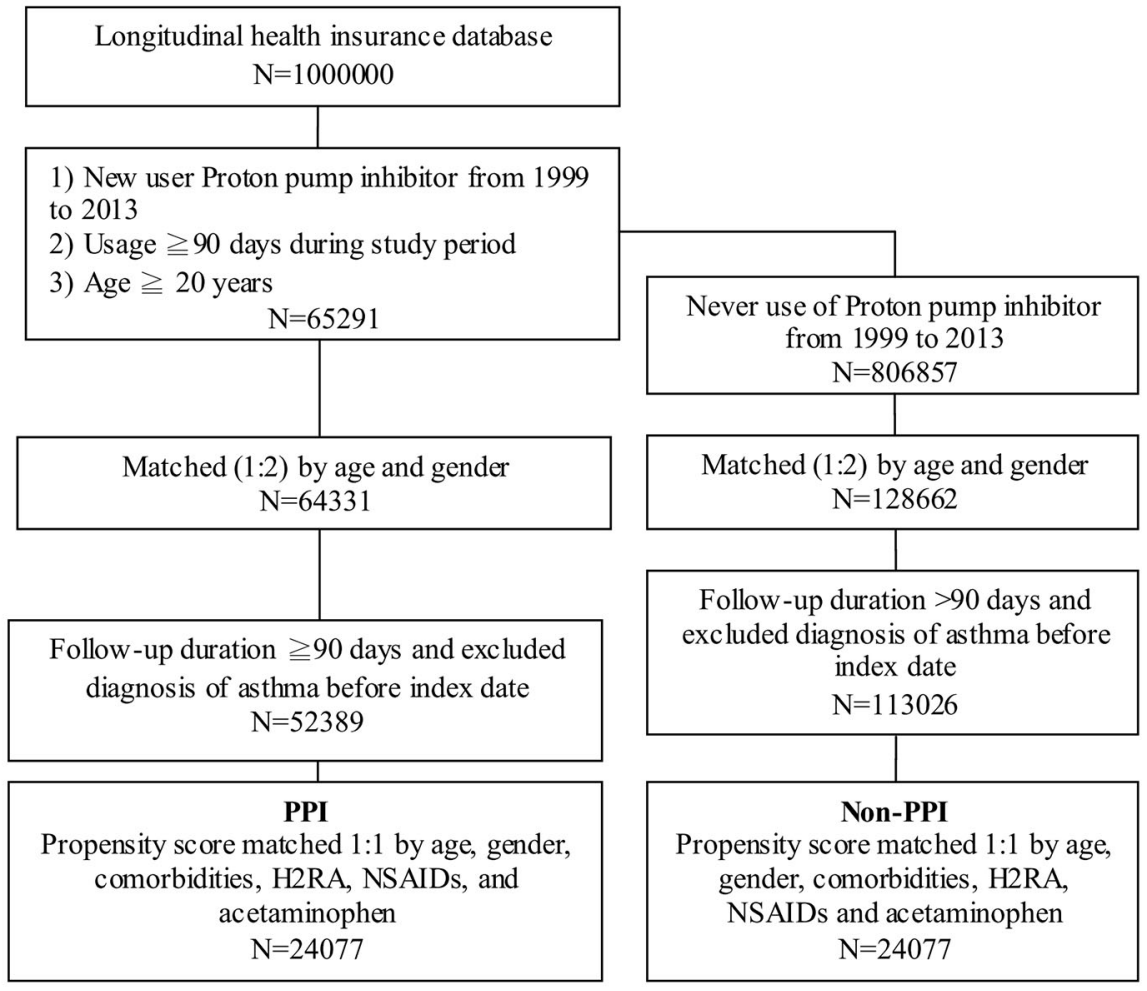

FIGURE 1 | Case selection flow chart of patient selection from one million nationally representative patients in the Taiwan National Health Insurance Research Database PPI, proton pump inhibitor; H2RA, histamine 2 receptor antagonists; NSAIDs, nonsteroidal anti-inflammatory drugs. 
analyses were performed using SPSS 18.0 (SPSS Inc., Chicago, IL, USA). A two-tailed P-value of 0.05 was considered statistically significant in this study.

\section{RESULTS}

The study cohorts consisted of 24,077 matched PPI patients and 24,077 matched non-PPI control subjects. The age and gender distributions were similar in both cohorts (Table 1). Most subjects were $40-65$ years of age $(53.8 \%$ of PPI and $53.3 \%$ of non-PPI). The mean ages of the PPI and non-PPI cohorts were $55.8 \pm 15.5$ and $56.7 \pm 15.1$ years, respectively.

The comorbidities, including allergic rhinitis, atopic dermatitis, GERD, peptic ulcer disease, hypertension, hyperlipidemia, diabetes, sleep apnea syndrome, and use of H2RA, NSAIDs, and acetaminophen, were significantly different between the unmatched PPI patients and the non-PPI controls ( $p$-values $<0.001)$, but no difference was observed between the matched PPI and non-PPI groups. The average follow-up period was $5.7 \pm 3.4$ years (137,582 person-years) for the PPI cohort and $6.9 \pm 3.5$ years $(165,323$ person-years) for the non-PPI cohort. Kaplan-Meier analysis showed that the cumulative probability of developing asthma by the end of the 14-year follow-up period was higher for the PPI cohort than for the non-PPI cohort (log-rank test, $p<0.001$; Figure 2).

Table 2 shows a total of 3,216 asthma events (1,823 for the PPI cohort and 1,393 for the non-PPI cohort). The incidence was 1.70fold higher (95\% CI, 1.59-1.83) in the PPI cohort than in the non-

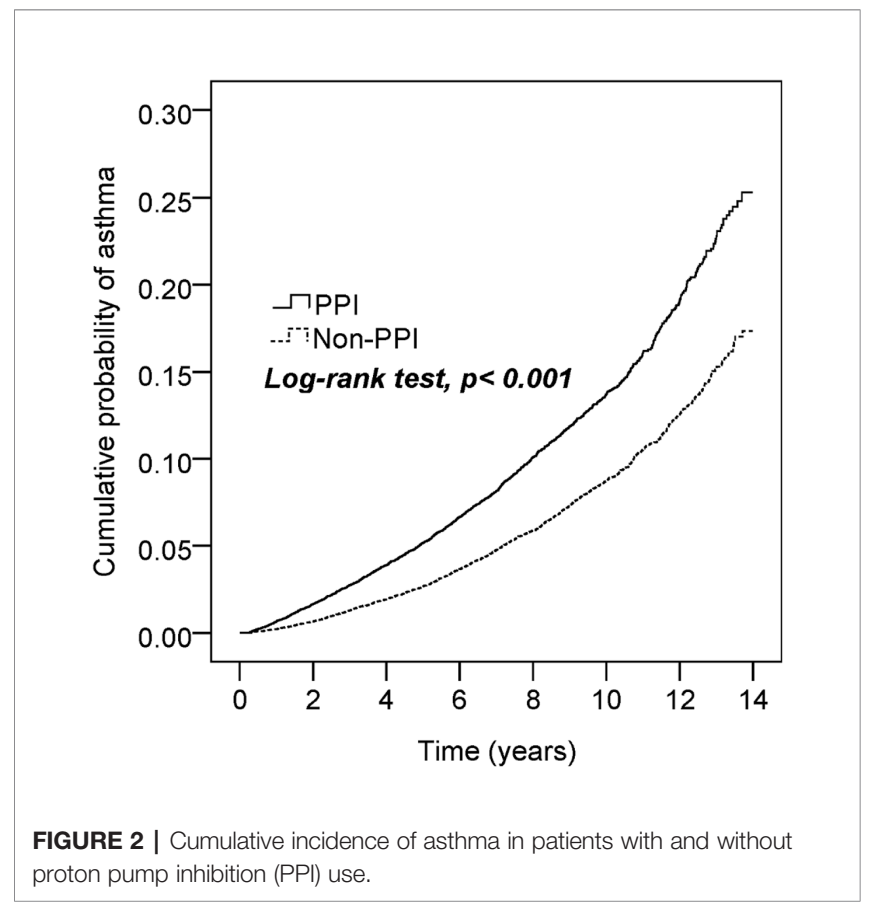

PPI cohort (13.3 vs. 8.4 per 1,000 person-years), with an adjusted HR of 1.76 (95\% CI, 1.64-1.88) after controlling for age, gender, comorbidities, and medications. The incidence of asthma decreased with age in both cohorts $(p=0.019)$, but the age-specific adjusted HRs for the PPI cohort, when compared with the non-PPI cohort,

TABLE 1 | Demographic characteristics, comorbidity, and medication in patients with asthma using or not using proton pump inhibitors (PPIs).

\begin{tabular}{|c|c|c|c|c|c|c|c|c|c|c|}
\hline & \multicolumn{4}{|c|}{ Unmatched } & \multirow[b]{3}{*}{$p$-value } & \multicolumn{4}{|c|}{ Matched } & \multirow[b]{3}{*}{ p-value } \\
\hline & \multicolumn{2}{|c|}{$\begin{array}{c}\text { PPI } \\
(\mathrm{N}=52,389)\end{array}$} & \multicolumn{2}{|c|}{$\begin{array}{c}\text { Non-PPI } \\
(\mathrm{N}=113,026)\end{array}$} & & \multicolumn{2}{|c|}{$\begin{array}{c}\text { PPI } \\
(\mathrm{N}=\mathbf{2 4 , 0 7 7 )}\end{array}$} & \multicolumn{2}{|c|}{$\begin{array}{c}\text { Non-PPI } \\
(\mathrm{N}=\mathbf{2 4 , 0 7 7 )}\end{array}$} & \\
\hline & $\mathbf{N}$ & $\%$ & n & $\%$ & & $n$ & $\%$ & n & $\%$ & \\
\hline Age & & & & & $0.006^{\star}$ & & & & & 0.168 \\
\hline $20-40$ & 9,390 & 17.9 & 19,774 & 17.5 & & 3,818 & 15.9 & 3,745 & 15.6 & \\
\hline $40-65$ & 28,632 & 54.7 & 61,485 & 54.4 & & 12,959 & 53.8 & 12,845 & 53.3 & \\
\hline$>=65$ & 14,367 & 27.4 & 31,767 & 28.1 & & 7,300 & 30.3 & 7,487 & 31.1 & \\
\hline Mean \pm SD & \multicolumn{2}{|c|}{$54.8 \pm 15.4$} & \multicolumn{2}{|c|}{$55.1 \pm 15.4$} & $0.001^{\star}$ & \multicolumn{2}{|c|}{$55.8 \pm 15.5$} & \multicolumn{2}{|c|}{$56.7 \pm 15.1$} & $<0.001^{\star}$ \\
\hline Gender & & & & & 0.076 & & & & & 0.784 \\
\hline Male & 23,705 & 45.2 & 51,670 & 45.7 & & 13,135 & 54.6 & 13,105 & 54.4 & \\
\hline Female & 28,684 & 54.8 & 61,356 & 54.3 & & 10,942 & 45.4 & 10,972 & 45.6 & \\
\hline Hypertension & 15,915 & 30.4 & 22,644 & 20.0 & $<0.001^{\star}$ & 7,429 & 30.9 & 7,533 & 31.3 & 0.306 \\
\hline Hyperlipidemia & 7,270 & 13.9 & 8,618 & 7.6 & $<0.001^{\star}$ & 3,171 & 13.2 & 3,247 & 13.5 & 0.308 \\
\hline GERD & 5,418 & 10.3 & 344 & 0.3 & $<0.001^{\star}$ & 341 & 1.4 & 344 & 1.4 & 0.908 \\
\hline Allergic rhinitis & 4,439 & 8.5 & 4,764 & 4.2 & $<0.001^{\star}$ & 1,740 & 7.2 & 1,802 & 7.5 & 0.279 \\
\hline Atopic dermatitis & 359 & 0.7 & 555 & 0.5 & $<0.001^{\star}$ & 157 & 0.7 & 159 & 0.7 & 0.910 \\
\hline Peptic ulcer disease & 26,648 & 50.9 & 3,333 & 2.9 & $<0.001^{\star}$ & 3,336 & 13.9 & 3,333 & 13.8 & 0.968 \\
\hline Diabetes & 8,549 & 16.3 & 9,774 & 8.6 & $<0.001^{\star}$ & 3,748 & 15.6 & 3,798 & 15.8 & 0.531 \\
\hline Sleep apnea syndrome & 3,209 & 6.1 & 2,983 & 2.6 & $<0.001^{\star}$ & 1,274 & 5.3 & 1,289 & 5.4 & 0.761 \\
\hline $\mathrm{H} 2 \mathrm{RA}$ & 19,240 & 36.7 & 6,228 & 5.5 & $<0.001^{\star}$ & 5,786 & 24.0 & 5,758 & 23.9 & 0.765 \\
\hline NSAIDs & 25,288 & 48.3 & 33,473 & 29.6 & $<0.001^{\star}$ & 10,818 & 44.9 & 11,003 & 45.7 & 0.090 \\
\hline Acetaminophen & 23,402 & 44.7 & 23,927 & 21.2 & $<0.001^{\star}$ & 9,476 & 39.4 & 9,625 & 40.0 & 0.165 \\
\hline
\end{tabular}

${ }^{*} p<0.01$.

PPI, proton pump inhibitor; GERD, gastroesophageal reflux disease; H2RA, histamine 2 receptor antagonists; NSAIDs, nonsteroidal anti-inflammatory drugs. 
TABLE 2 | Incidence of asthma and the Cox proportional hazard model estimated proton pump inhibitor (PPI) cohort to non-PPI cohort hazard ratio.

\begin{tabular}{|c|c|c|c|c|c|c|c|c|}
\hline & \multicolumn{3}{|c|}{ PPI } & \multicolumn{3}{|c|}{ Non-PPI } & \multirow[b]{2}{*}{ Crude HR (95\% C.I.) } & \multirow[b]{2}{*}{ Adjusted HR (95\% C.I.) } \\
\hline & Asthma event & PY & rate & Asthma event & PY & rate & & \\
\hline Total & 1,823 & 137,582 & 13.3 & 1,393 & 165,323 & 8.4 & $1.70(1.59-1.83)$ & $1.76(1.64-1.88)$ \\
\hline \multicolumn{9}{|l|}{ Age } \\
\hline $20-40$ & 196 & 23,859 & 8.2 & 113 & 27,634 & 4.1 & $2.21(1.75-2.79)$ & $2.22(1.76-2.80)$ \\
\hline $40-65$ & 891 & 77,631 & 11.5 & 643 & 91,818 & 7.0 & $1.81(1.63-2.00)$ & 1.83 (1.66-2.03) \\
\hline$>=65$ & 736 & 36,091 & 20.4 & 637 & 45,870 & 13.9 & $1.56(1.41-1.74)$ & $\begin{array}{c}1.60(1.44-1.78) \\
\text { p for interaction }=0.019\end{array}$ \\
\hline \multicolumn{9}{|l|}{ Gender } \\
\hline Male & 894 & 75,156 & 11.9 & 721 & 90,282 & 8.0 & $1.61(1.46-1.78)$ & 1.65 (1.50-1.83) \\
\hline Female & 929 & 62,426 & 14.9 & 672 & 75,041 & 9.0 & $1.80(1.63-1.99)$ & $\begin{array}{c}1.86(1.69-2.06) \\
p \text { for interaction= } 0.127\end{array}$ \\
\hline \multicolumn{9}{|c|}{ Hypertension } \\
\hline No & 1,187 & 100,312 & 11.8 & 839 & 119,430 & 7.0 & 1.86 (1.70-2.03) & $1.90(1.74-2.08)$ \\
\hline Yes & 636 & 37,270 & 17.1 & 554 & 45,893 & 12.1 & $1.50(1.34-1.68)$ & $\begin{array}{c}1.55(1.38-1.74) \\
\text { p for interaction }=0.009\end{array}$ \\
\hline \multicolumn{9}{|c|}{ Hyperlipidemia } \\
\hline No & 1,590 & 121,675 & 13.1 & 1,206 & 146,560 & 8.2 & $1.73(1.60-1.86)$ & 1.78 (1.65-1.92) \\
\hline Yes & 233 & 15,908 & 14.6 & 187 & 18,763 & 10.0 & $1.54(1.27-1.87)$ & $\begin{array}{c}1.60(1.32-1.94) \\
\text { p for interaction }=0.354\end{array}$ \\
\hline \multicolumn{9}{|l|}{ GERD } \\
\hline No & 1,803 & 136,289 & 13.2 & 1,380 & 163,997 & 8.4 & 1.70 (1.59-1.83) & 1.76 (1.64-1.89) \\
\hline Yes & 20 & 1,293 & 15.5 & 13 & 1,326 & 9.8 & $1.57(0.78-3.16)$ & $\begin{array}{c}1.57(0.77-3.20) \\
\text { p for interaction }=0.786\end{array}$ \\
\hline \multicolumn{9}{|c|}{ Allergic rhinitis } \\
\hline No & 1,652 & 128,132 & 12.9 & 1,236 & 153,854 & 8.0 & $1.74(1.62-1.87)$ & $1.80(1.67-1.94)$ \\
\hline Yes & 171 & 9,450 & 18.1 & 157 & 11,469 & 13.7 & $1.41(1.14-1.75)$ & $\begin{array}{c}1.44(1.15-1.79) \\
\text { p for interaction }=0.065\end{array}$ \\
\hline \multicolumn{9}{|c|}{ Atopic dermatitis } \\
\hline No & 1,806 & 136,762 & 13.2 & 1,380 & 164,317 & 8.4 & 1.70 (1.59-1.83) & 1.80 (1.68-1.93) \\
\hline Yes & 17 & 820 & 20.7 & 13 & 1,006 & 12.9 & $1.70(0.82-3.50)$ & $\begin{array}{c}1.76(0.84-3.69) \\
\text { p for interaction }=0.908\end{array}$ \\
\hline \multicolumn{9}{|c|}{ Peptic ulcer disease } \\
\hline No & 1,521 & 117,586 & 12.9 & 1,089 & 143,531 & 7.6 & 1.87 (1.73-2.02) & $1.95(1.80-2.11)$ \\
\hline Yes & 302 & 19,996 & 15.1 & 304 & 21,792 & 13.9 & $1.11(0.95-1.30)$ & $\begin{array}{r}1.14(0.97-1.34) \\
\text { p for interaction }<0.001\end{array}$ \\
\hline \multicolumn{9}{|l|}{ Diabetes } \\
\hline No & 1,547 & 120,310 & 12.9 & 1,163 & 142,852 & 8.1 & $1.72(1.59-1.85)$ & $1.76(1.63-1.90)$ \\
\hline Yes & 276 & 17,272 & 16.0 & 230 & 22,471 & 10.2 & $1.66(1.39-1.97)$ & $\begin{array}{r}1.72(1.44-2.05) \\
p \text { for interaction }=0.943\end{array}$ \\
\hline \multicolumn{9}{|c|}{ Sleep apnea syndrome } \\
\hline No & 1,722 & 131,137 & 13.1 & 1,298 & 157,665 & 8.2 & $1.73(1.61-1.86)$ & 1.78 (1.66-1.92) \\
\hline Yes & 101 & 6,445 & 15.7 & 95 & 7,658 & 12.4 & $1.32(0.997-1.75)$ & $\begin{array}{r}1.33(1.002-1.76) \\
p \text { for interaction }=0.074\end{array}$ \\
\hline \multicolumn{9}{|l|}{ H2RA } \\
\hline No & 1,292 & 97,235 & 13.3 & 983 & 119,937 & 8.2 & $1.75(1.61-1.90)$ & $1.81(1.66-1.96)$ \\
\hline Yes & 531 & 40,347 & 13.2 & 410 & 45,386 & 9.0 & $1.56(1.37-1.77)$ & $\begin{array}{r}1.58(1.38-1.79) \\
p \text { for interaction }=0.029\end{array}$ \\
\hline \multicolumn{9}{|l|}{ NSAIDs } \\
\hline No & 803 & 61,456 & 13.1 & 560 & 75,846 & 7.4 & $1.84(1.65-2.05)$ & $1.93(1.73-2.15)$ \\
\hline Yes & 1,020 & 76,126 & 13.4 & 833 & 89,477 & 9.3 & $1.58(1.44-1.73)$ & $\begin{array}{r}1.61(1.47-1.76) \\
\text { p for interaction }<0.001\end{array}$ \\
\hline \multicolumn{9}{|c|}{ Acetaminophen } \\
\hline No & 931 & 72,357 & 12.9 & 626 & 85,788 & 7.3 & $1.82(1.65-2.02)$ & $1.88(1.70-2.08)$ \\
\hline Yes & 892 & 65,225 & 13.7 & 767 & 79,535 & 9.6 & $1.58(1.44-1.74)$ & $\begin{array}{r}1.64(1.49-1.81) \\
p \text { for interaction }=0.001\end{array}$ \\
\hline
\end{tabular}

PY, Person-years; Rate, 1/1,000 Person-Years; PPI, proton pump inhibitor; GERD, gastroesophageal reflux disease; H2RA, histamine 2 receptor antagonists; NSAIDs, nonsteroidal anti-inflammatory drugs.

were statistically significant for all age groups. Among the analysis of the listed comorbidities, we found that the asthma prevalence was higher for patients without peptic ulcer disease in the PPI cohort (adjusted HR of 1.95; 95\% CI, 1.80-2.11) but did not differ in patients with peptic ulcer disease (adjusted HR of 1.14; 95\% CI, 0.97-1.34). Patients not using H2RA before PPIs had a higher incidence of asthma (adjusted HR of 1.81; 95\% CI, 1.66-1.96) than in those cases who had used H2RA (adjusted HR of 1.58; 95\% CI, 
TABLE 3 | Cox proportional hazard model estimated proton pump inhibitor (PPI) hazard ratio among different PPI types.

\begin{tabular}{|c|c|c|c|c|c|c|}
\hline & $\mathbf{N}$ & No. of asthma event & Crude HR & 95\% C.I. & Adjusted $\mathrm{HR}^{\dagger}$ & 95\% C.I. \\
\hline Esomeprazole & 12,807 & 1,071 & $1.27^{\star}$ & $1.17-1.38$ & $1.26^{*}$ & $1.15-1.37$ \\
\hline Lansoprazole & 13,842 & 1,187 & $1.18^{*}$ & $1.08-1.28$ & $1.23^{*}$ & $1.13-1.34$ \\
\hline Omeprazole & 10,530 & 1,054 & $1.36^{*}$ & $1.25-1.49$ & $1.38^{*}$ & $1.26-1.50$ \\
\hline Pantoprazole & 10,131 & 841 & $1.15^{\star}$ & $1.05-1.26$ & $1.13^{*}$ & $1.04-1.24$ \\
\hline Rabeprazole & 5,705 & 448 & 1.01 & $0.91-1.12$ & 1.04 & $0.94-1.15$ \\
\hline
\end{tabular}

${ }^{\dagger}$ Adjusted for age, gender, hypertension, hyperlipidemia, GERD, allergic rhinitis, atopic dermatitis, peptic ulcer disease, diabetes, sleep apnea syndrome, H2RA, NSAIDs, and acetaminophen.; ${ }^{*} p<0.01$.

1.38-1.79). If the patients were not exposed to asthma-related medications (NSAIDs and acetaminophen), the asthma risks due to PPI use were also higher (adjusted HR of 1.93; 95\% CI, 1.73-2.15 in the NSAIDs cohort and 1.88; 95\% CI, 1.70-2.08 in the acetaminophen cohort).

Table 3 shows the results of the Cox proportional hazard model estimated PPI hazard ratio among the different PPI types. Esomeprazole, lansoprazole, omeprazole, and pantoprazole were significantly related to asthma risk, but rabeprazole was not (adjusted HR of 1.04; 95\% CI, 0.94-1.15).

\section{DISCUSSION}

To our knowledge, this is the first study to evaluate the relationship between PPI use and the subsequent risk of asthma. We identified a significant risk of asthma among patients using PPIs when compared to the non-PPI population after matching by propensity score and adjusting for age, gender, comorbidities, and asthma medications. The incidence of asthma was higher in patients not using H2RA before PPIs than in those cases who had used H2RA. Patients not exposed to asthmarelated medications (NSAIDs and acetaminophen) also had a higher asthma risk due to PPIs (adjusted HR of 1.93 in the NSAIDs cohort and 1.88 in the acetaminophen cohort).

Many studies have proved that some medications, like aspirin, NSAIDs, and acetaminophen, may be related to the risk of asthma. The use of acetaminophen may represent an important risk factor for the development and/or maintenance of asthma in adolescent children (Beasley et al., 2011). For example, paracetamol exposure in pregnancy and early infancy has been considered to have an association with the subsequent development of childhood asthma (age $\geq 5$ years) (Cheelo et al., 2015). A previous retrospective cohort study of the Taiwan NHIRD indicated that short-term aspirin, ibuprofen, and diclofenac use was probably correlated with asthma exacerbation in asthmatic children (Lo et al., 2016). However, none of these studies mentioned any possible association of acidsuppressive therapy and asthma risk. The fully adjusted Cox regression model presented in our study for competing risk analysis (i.e., age, gender, allergic rhinitis, atopic dermatitis, GERD, peptic ulcer disease, hypertension, hyperlipidemia, diabetes, sleep apnea syndrome, and use of H2RA, NSAIDs, and acetaminophen) suggests that PPIs may play an independent role in the development of asthma.

PPIs inhibit the $\mathrm{H}^{+}-\mathrm{K}^{+}$-ATPase, which is the final step of gastric acid secretion by parietal cells. Since the 1980s, when the first generation of PPIs was made available, PPIs have become the most commonly used medications for acid suppression (Forgacs and Loganayagam, 2008). PPIs are the first-line antisecretory therapy in the treatment of peptic ulcer disease and are indicated as a treatment for GERD, including erosive esophagitis, and as maintenance therapy in patients with severe erosive esophagitis or Barrett's esophagus (Wolfe and Sachs, 2000). PPIs are also effective in preventing NSAID-induced gastroduodenal toxicity and in healing gastroduodenal ulcers associated with NSAIDs when NSAIDS cannot be discontinued (Agrawal et al., 2000). In the COGENT trial, prophylactic use of a PPI reduced the rate of upper gastrointestinal bleeding among patients receiving aspirin and clopidogrel (Bhatt et al., 2010).

In recent years, increasing numbers of studies have suggested an association between PPIs and an increased risk of adverse health outcomes. Some studies have shown that PPIs are associated with a significant rise in acute interstitial nephritis (Blank et al., 2014), chronic kidney disease (CKD) (Lazarus et al., 2016), kidney disease progression, and end-stage renal disease (Xie et al., 2017). A large prospective observational German cohort study showed that patients receiving PPIs had a higher risk of incident dementia (Gomm et al., 2016). PPI use has been associated with an increased risk of incident and recurrent Clostridium difficile infections (Kwok et al., 2012), communityacquired pneumonia (Filion et al., 2014), and hospital-associated pneumonia (Herzig et al., 2009). A relationship between cardiovascular events and PPI use has also been mentioned (Melloni et al., 2015). A longitudinal observational cohort study of United States veterans also found an increased risk of death among users of PPIs (Xie et al., 2017). Our study found that PPI use is an independent risk for asthma.

Aggressive acid suppressive therapy with PPIs has been recommended to improve asthma outcomes in asthmatics with GERD. A study of 30 nonsmoking adult asthmatics with GERD found that a 3-month regimen of acid suppressive therapy with omeprazole improved asthma symptoms and pulmonary function in $73 \%$ of the subjects (Harding et al., 1996). Two placebocontrolled trials that investigated the efficacy of PPI on GERDrelated chronic cough indicated that PPI treatment relieves GERDrelated chronic cough but recommended using a double-standard dose of the PPI for a minimum of 2 to 3 months (Harding, 2003). However, a parallel-group, double-blind trial of 412 participants with asthma inadequately controlled by inhaled corticosteroids and with minimal or no symptoms of GERD showed no improvement in asthma outcomes by a treatment with $40 \mathrm{mg}$ esomeprazole twice a day; these findings indicated that asymptomatic GERD might not 
be a possible cause of poorly controlled asthma (Mastronarde et al., 2009).

A review study identified 13 publications from 1989 to 2012 related to treatment of asymptomatic GERD in school-age children with asthma poorly controlled by inhaled corticosteroids. The FDA-approved doses of PPIs did not improve their asthma outcomes (Blake and Teague, 2013); consequently, the authors commented that GERD and asthma may be associated by chance alone, because GERD is highly prevalent in the general population (Dent et al., 2005).

PPIs provide a stronger acid suppression when compared to H2RA, so they result in a faster control of peptic ulcer disease symptoms and higher ulcer healing rates (Walan et al., 1989). A meta-analysis that enrolled fourteen trials and a total of 1,720 patients concluded that PPIs were more effective than H2RA at reducing clinically important and overt upper gastrointestinal bleeding. No differences were noted between PPIs and H2RA in the risk of nosocomial pneumonia, ICU mortality, or ICU length of stay (Alhazzani et al., 2013). However, a decision-analytic model aimed at determining the cost effectiveness of stress ulcer prophylaxis with H2RA versus PPIs in critically ill and mechanically ventilated adults from a health care institutional perspective concluded that providing stress ulcer prophylaxis with H2RA therapy may reduce costs, increase survival, and avoid complications when compared with PPI therapy (Hammond et al., 2017).

A recent multicenter retrospective study examined the effect of preventing clinically important GI bleeding (CIGIB) with prophylactic PPIs or H2RA among critically ill adults with at least one stress ulcer risk factor in ICU care in US nonfederal hospitals. They found the hazard ratio for CIGIB was two times greater for PPIs patients than for H2RA patients (adjusted HR 1.82 [95\% CI, 1.19-2.78]) (Lilly et al., 2018). H2RA administration has been associated with many rare side effects (cardiac arrhythmia; cardiac arrest occurring with rapid infusion (Hinrichsen et al., 1995; Lee et al., 2004); increases in serum creatinine observed with cimetidine; immune-mediated interstitial nephritis; both cholestatic and hepatocellular injury (Fisher and Le Couteur, 2001); CNS side effects including confusion, restlessness, somnolence, agitation, headaches, dizziness, and hallucinations and seizures with prolonged therapy (Cantu and Korek, 1991); and B12 deficiency with long-term H2RA use (Lam et al., 2013); however, many of these adverse effects are rapidly reversible or resolve after withdrawal of the drug (Fisher and Le Couteur, 2001). In our study, we found a lower PPI-related asthma risk if the patients used the H2RA first, before PPIs use.

Some past studies have found an association between GERD and asthma; however, no causal link between GERD and asthma was evident in our study. Conversely, we found that PPIs, rather than GERD, influence the subsequent occurrence of asthma. Our results showed that most PPIs were associated with asthma risk; the exception was rabeprazole. Our smaller patient sample size is a likely explanation, but further study could be considered to evaluate this difference.

Some limitations of this study should be considered. First, we could not establish a causal relationship between PPIs and asthma risk based on this retrospective cohort study. A prospective double blind study would be more convincing. Second, we found a higher incidence of asthma for the patients without peptic ulcer or exposure to asthma-related medications (NSAIDs and acetaminophen) in the PPI group than in the nonPPI group. A well-designed randomized control trial is necessary to confirm the higher asthma risk of PPIs, but PPIs are more popular regimens clinically due to their strong antacid effects and relatively low cost. Third, we noticed that rabeprazole did not increase the asthma risk, in contrast to the other PPIs (esomeprazole, lansoprazole, omeprazole, and pantoprazole). We are unable to explain this difference, but it might reflect the lower use of rabeprazole in our study population or, indeed, a pharmacological difference.

\section{CONCLUSIONS}

Our data suggest that patients with long-time PPI use have a significantly higher risk of developing asthma when compared with the general population, regardless of gender and age. We recommended H2RA use prior to PPIs in patients who have no underlying peptic ulcers. The pathophysiological association between PPIs and asthma needs further investigation.

\section{DATA AVAILABILITY STATEMENT}

All datasets generated for this study are included in the article/ supplementary material.

\section{ETHICS STATEMENT}

The studies involving human participants were reviewed and approved by institutional review board of Chung Shan Medical University Hospital. Written informed consent for participation was not required for this study in accordance with the national legislation and the institutional requirements.

\section{AUTHOR CONTRIBUTIONS}

Conception and design: Y-TW and JW. Administrative support: JW. Data collection and organization: All authors. Data analysis and interpretation: All authors. Manuscript writing: All authors. Final approval of the manuscript: All authors.

\section{FUNDING}

This work was supported by grants from Chung Shan Medical University Hospital research program, Taichung, Taiwan (CSH2018-C-023). 


\section{REFERENCES}

Agrawal, N. M., Campbell, D. R., Safdi, M. A., Lukasik, N. L., Huang, B., and Haber, M. M. (2000). Superiority of lansoprazole vs ranitidine in healing nonsteroidal antiinflammatory drug-associated gastric ulcers: results of a double-blind, randomized, multicenter study. NSAID-Associated Gastric Ulcer Study Group. Arch. Intern. Med. 160, 1455-1461. doi: 10.1001/archinte.160.10.1455

Alhazzani, W., Alenezi, F., Jaeschke, R. Z., Moayyedi, P., and Cook, D. J. (2013). Proton pump inhibitors versus histamine 2 receptor antagonists for stress ulcer prophylaxis in critically ill patients: a systematic review and meta-analysis. Crit. Care Med. 41, 693-705. doi: 10.1097/CCM.0b013e3182758734

Beasley, R. W., Clayton, T. O., Crane, J., Lai, C. K., Montefort, S. R., Mutius, E., et al. (2011). Acetaminophen use and risk of asthma, rhinoconjunctivitis, and eczema in adolescents: International Study of Asthma and Allergies in Childhood Phase Three. Am. J. Respir. Crit. Care Med. 183, 171-178. doi: 10.1164/rccm.201005-0757OC

Bhatt, D. L., Cryer, B. L., Contant, C. F., Cohen, M., Lanas, A., Schnitzer, T. J., et al. (2010). Clopidogrel with or without omeprazole in coronary artery disease. $N$. Engl. J. Med. 363, 1909-1917. doi: 10.1056/NEJMoa1007964

Blake, K., and Teague, W. G. (2013). Gastroesophageal reflux disease and childhood asthma. Curr. Opin. Pulm. Med. 19, 24-29. doi: 10.1097/ MCP.0b013e32835b582b

Blank, M. L., Parkin, L., Paul, C., and Herbison, P. (2014). A nationwide nested case-control study indicates an increased risk of acute interstitial nephritis with proton pump inhibitor use. Kidney Int. 86, 837-844. doi: 10.1038/ki.2014.74

Bor, S., Kitapcioglu, G., Solak, Z. A., Ertilav, M., and Erdinc, M. (2010). Prevalence of gastroesophageal reflux disease in patients with asthma and chronic obstructive pulmonary disease. J. Gastroenterol. Hepatol. 25, 309-313. doi: 10.1111/j.1440-1746.2009.06035.x

Broers, C., Tack, J., and Pauwels, A. (2018). Review article: gastro-oesophageal reflux disease in asthma and chronic obstructive pulmonary disease. Aliment Pharmacol. Ther. 47, 176-191. doi: 10.1111/apt.14416

Cantu, T. G., and Korek, J. S. (1991). Central nervous system reactions to histamine-2 receptor blockers. Ann. Intern. Med. 114, 1027-1034. doi: 10.7326/0003-4819-114-12-1027

Cheelo, M., Lodge, C. J., Dharmage, S. C., Simpson, J. A., Matheson, M., Heinrich, J., et al. (2015). Paracetamol exposure in pregnancy and early childhood and development of childhood asthma: a systematic review and meta-analysis. Arch. Dis. Child 100, 81-89. doi: 10.1136/archdischild-2012-303043

Chipps, B. E., Haselkorn, T., Paknis, B., Ortiz, B., Bleecker, E. R., Kianifard, F., et al. (2018). More than a decade follow-up in patients with severe or difficult-totreat asthma: The Epidemiology and Natural History of Asthma: Outcomes and Treatment Regimens (TENOR) II. J. Allergy Clin. Immunol. 141, 15901597.e1599. doi: 10.1016/j.jaci.2017.07.014

Dent, J., El-Serag, H. B., Wallander, M. A., and Johansson, S. (2005). Epidemiology of gastro-oesophageal reflux disease: a systematic review. Gut 54, 710-717. doi: 10.1136/gut.2004.051821

Depla, A. C., Bartelsman, J. F., Roos, C. M., Tytgat, G. N., and Jansen, H. M. (1988). Beneficial effect of omeprazole in a patient with severe bronchial asthma and gastro-oesophageal reflux. Eur. Respir. J. 1, 966-968.

Filion, K. B., Chateau, D., Targownik, L. E., Gershon, A., Durand, M., Tamim, H., et al. (2014). Proton pump inhibitors and the risk of hospitalisation for community-acquired pneumonia: replicated cohort studies with metaanalysis. Gut 63, 552-558. doi: 10.1136/gutjnl-2013-304738

Fisher, A. A., and Le Couteur, D. G. (2001). Nephrotoxicity and hepatotoxicity of histamine H2 receptor antagonists. Drug Saf. 24, 39-57. doi: 10.2165/ 00002018-200124010-00004

Forgacs, I., and Loganayagam, A. (2008). Overprescribing proton pump inhibitors. Bmj 336, 2-3. doi: 10.1136/bmj.39406.449456.BE

Gomm, W., Von Holt, K., Thome, F., Broich, K., Maier, W., Fink, A., et al. (2016). Association of Proton Pump Inhibitors With Risk of Dementia: A Pharmacoepidemiological Claims Data Analysis. JAMA Neurol. 73, 410-416. doi: 10.1001/jamaneurol.2015.4791

Hammond, D. A., Kathe, N., Shah, A., and Martin, B. C. (2017). Cost-Effectiveness of Histamine2 Receptor Antagonists Versus Proton Pump Inhibitors for Stress Ulcer Prophylaxis in Critically Ill Patients. Pharmacotherapy 37, 43-53. doi: 10.1002/phar.1859
Harding, S. M., Richter, J. E., Guzzo, M. R., Schan, C. A., Alexander, R. W., and Bradley, L. A. (1996). Asthma and gastroesophageal reflux: acid suppressive therapy improves asthma outcome. Am. J. Med. 100, 395-405. doi: 10.1016/ S0002-9343(97)89514-9

Harding, S. M. (2003). Recent clinical investigations examining the association of asthma and gastroesophageal reflux. Am. J. Med. 115 (Suppl 3A), 39s-44s. doi: 10.1016/S0002-9343(03)00191-8

Herzig, S. J., Howell, M. D., Ngo, L. H., and Marcantonio, E. R. (2009). Acidsuppressive medication use and the risk for hospital-acquired pneumonia. Jama 301, 2120-2128. doi: 10.1001/jama.2009.722

Hinrichsen, H., Halabi, A., and Kirch, W. (1995). Clinical aspects of cardiovascular effects of H2-receptor antagonists. Eur. J. Clin. Invest. 25 Suppl 1, 47-56. doi: 10.1002/j.1552-4604.1995.tb04998.x

Irwin, R. S., Baumann, M. H., Bolser, D. C., Boulet, L. P., Braman, S. S., Brightling, C. E., et al. (2006). Diagnosis and management of cough executive summary: ACCP evidence-based clinical practice guidelines. Chest 129, 1s-23s. doi: 10.1378/chest.129.1_suppl.1S

Kahrilas, P. J., Altman, K. W., Chang, A. B., Field, S. K., Harding, S. M., Lane, A. P., et al. (2016). Chronic Cough Due to Gastroesophageal Reflux in Adults: CHEST Guideline and Expert Panel Report. Chest 150, 1341-1360. doi: 10.1016/j.chest.2016.08.1458

Kastelik, J. A., Aziz, I., Ojoo, J. C., Thompson, R. H., Redington, A. E., and Morice, A. H. (2005). Investigation and management of chronic cough using a probability-based algorithm. Eur. Respir. J. 25, 235-243. doi: 10.1183/ 09031936.05.00140803

Kwok, C. S., Arthur, A. K., Anibueze, C. I., Singh, S., Cavallazzi, R., and Loke, Y. K. (2012). Risk of Clostridium difficile infection with acid suppressing drugs and antibiotics: meta-analysis. Am. J. Gastroenterol. 107, 1011-1019. doi: 10.1038/ ajg. 2012.108

Lam, J. R., Schneider, J. L., Zhao, W., and Corley, D. A. (2013). Proton pump inhibitor and histamine 2 receptor antagonist use and vitamin B12 deficiency. Jama 310, 2435-2442. doi: 10.1001/jama.2013.280490

Lazarus, B., Chen, Y., Wilson, F. P., Sang, Y., Chang, A. R., Coresh, J., et al. (2016). Proton Pump Inhibitor Use and the Risk of Chronic Kidney Disease. JAMA Intern. Med. 176, 238-246. doi: 10.1001/jamainternmed.2015.7193

Lee, K. W., Kayser, S. R., Hongo, R. H., Tseng, Z. H., and Scheinman, M. M. (2004). Famotidine and long QT syndrome. Am. J. Cardiol. 93, 1325-1327. doi: 10.1016/j.amjcard.2004.02.025

Lee, Y. C., Huang, Y. T., Tsai, Y. W., Huang, S. M., Kuo, K. N., Mckee, M., et al. (2010). The impact of universal National Health Insurance on population health: the experience of Taiwan. BMC Health Serv. Res. 10, 225. doi: 10.1186/ 1472-6963-10-225

Lilly, C. M., Aljawadi, M., Badawi, O., Onukwugha, E., Tom, S. E., Magder, L. S., et al. (2018). Comparative Effectiveness of Proton Pump Inhibitors vs Histamine Type 2 Receptor Blockers for Preventing Clinically Important Gastrointestinal Bleeding During Intensive Care: A Population-Based Study. Chest 154, 557-566. doi: 10.1016/j.chest.2018.05.015

Lo, P. C., Tsai, Y. T., Lin, S. K., and Lai, J. N. (2016). Risk of asthma exacerbation associated with nonsteroidal anti-inflammatory drugs in childhood asthma: A nationwide population-based cohort study in Taiwan. Med. (Baltimore) 95, e5109. doi: 10.1097/MD.0000000000005109

Mastronarde, J. G., Anthonisen, N. R., Castro, M., Holbrook, J. T., Leone, F. T., Teague, W. G., et al. (2009). Efficacy of esomeprazole for treatment of poorly controlled asthma. N. Engl. J. Med. 360, 1487-1499. doi: 10.1056/ NEJMoa0806290

Meier, J. H., Mcnally, P. R., Punja, M., Freeman, S. R., Sudduth, R. H., Stocker, N., et al. (1994). Does omeprazole (Prilosec) improve respiratory function in asthmatics with gastroesophageal reflux? A double-blind, placebo-controlled crossover study. Dig. Dis. Sci. 39, 2127-2133. doi: 10.1007/BF02090360

Melloni, C., Washam, J. B., Jones, W. S., Halim, S. A., Hasselblad, V., Mayer, S. B., et al. (2015). Conflicting results between randomized trials and observational studies on the impact of proton pump inhibitors on cardiovascular events when coadministered with dual antiplatelet therapy: systematic review. Circ. Cardiovasc. Qual. Outcomes 8, 47-55. doi: 10.1161/CIRCOUTCOMES 114.001177

Pope, ,. C. E.2nd (1994). Acid-reflux disorders. N. Engl. J. Med. 331, 656-660. doi: 10.1056/NEJM199409083311007 
Ravelli, A. M., Panarotto, M. B., Verdoni, L., Consolati, V., and Bolognini, S. (2006). Pulmonary aspiration shown by scintigraphy in gastroesophageal reflux-related respiratory disease. Chest 130, 1520-1526. doi: 10.1378/chest.130.5.1520

Walan, A., Bader, J. P., Classen, M., Lamers, C. B., Piper, D. W., Rutgersson, K., et al. (1989). Effect of omeprazole and ranitidine on ulcer healing and relapse rates in patients with benign gastric ulcer. N. Engl. J. Med. 320, 69-75. doi: 10.1056/NEJM198901123200201

Wolfe, M. M., and Sachs, G. (2000). Acid suppression: optimizing therapy for gastroduodenal ulcer healing, gastroesophageal reflux disease, and stressrelated erosive syndrome. Gastroenterology 118, S9-31. doi: 10.1016/S00165085(00)70004-7

Xie, Y., Bowe, B., Li, T., Xian, H., Yan, Y., and Al-Aly, Z. (2017). Risk of death among users of Proton Pump Inhibitors: a longitudinal observational cohort study of United States veterans. BMJ Open 7, e015735. doi: 10.1136/bmjopen2016-015735

Conflict of Interest: The authors declare that the research was conducted in the absence of any commercial or financial relationships that could be construed as a potential conflict of interest.

Copyright (c) 2020 Wang, Tsai, Wang and Wei. This is an open-access article distributed under the terms of the Creative Commons Attribution License (CC BY). The use, distribution or reproduction in other forums is permitted, provided the original author(s) and the copyright owner(s) are credited and that the original publication in this journal is cited, in accordance with accepted academic practice. No use, distribution or reproduction is permitted which does not comply with these terms. 